\section{Kidney \\ Blood Pressure Research}

Kidney Blood Press Res 2013;37:103-115

\title{
The Effects of Arterial Blood Pressure Reduction on Endocan and Soluble Endothelial Cell Adhesion Molecules (CAMs) and CAMs Ligands Expression in Hypertensive Patients on Ca-Channel Blocker Therapy
}

\author{
Refmir Tadzic ${ }^{a}$ Martina Mihalj ${ }^{b} \quad$ Aleksandar Vceved Johann Ennen ${ }^{\mathrm{e}}$ \\ Arijan Tadzic ${ }^{f}$ Ines Drenjancevic ${ }^{b}$
}

\begin{abstract}
${ }^{a}$ Gesundheitszentrum Lange Reihe Dr. Tadzic und Kollegen, Hamburg, Germany; bUniversity Josip Juraj Strossmayer of Osijek, Faculty of Medicine Osijek, Dept of Physiology and Immunology, Osijek, Croatia; ' University Josip Juraj Strossmayer of Osijek, Faculty of Medicine Osijek, Dept of Internal Medicine, History of Medicine and Medical Ethics, Osijek, Croatia; ${ }^{\mathrm{d} C l i n i c a l ~ H o s p i t a l ~ C e n t e r ~ O s i j e k, ~}$

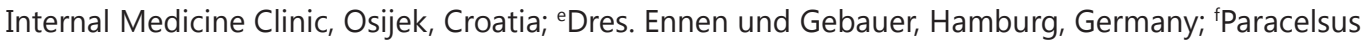
Medizinische Privatuniversität, Salzburg, Österreich
\end{abstract}

Key Words

sCAMs • CD11a/LFA-1 • CD15 • Hypertension • Endocan

\begin{abstract}
Background/Aims: To determine the effect of arterial blood pressure (BP) reduction on endocan and soluble cell adhesion molecules' (sCAM) plasma concentration and expression of their ligands on circulatory leukocyte subpopulations. Methods: 24 hypertensive subjects of both sexes (age: $53 \pm 8$ yrs) were treated with Ca-channel blocker, amlodipin (5-10 mg/ day for 8 weeks; to reach $B P \leq 139 / 89 \mathrm{mmHg}$ ). The serum sCAMs and endocan concentrations were determined by ELISA kits. Level of ICAM/VCAM ligands on leukocytes was assessed by flow cytometry. Paired t-test, or t-test were used as appropriate, with Pearson's correlation calculated; $\mathrm{p}<0.05$ was considered significant (SigmaPlot v.11). Results: sICAM-1 and sVCAM-1 were decreased ( $p \leq 0.001$ and $p=0.002$, respectively), while E-selectin concentration was increased after amlodipin treatment $(P=0.014)$. CD11a/LFA-1 (ICAM-1 and endocan ligand) was significantly increased in all three cell types with BP decrease. CD15 and CD49d/ VLA-4 (VCAM-1 ligand) did not change after the treatment. There was significant positive correlation of systolic and diastolic BP with ICAM-1 and VCAM-1, and significant negative correlation of systolic BP with CD11a/LFA-1. Endocan significantly positively correlated with
\end{abstract}




\section{Kidney Blood Pressure Research}

Kidney Blood Press Res 2013;37:103-115

\begin{tabular}{l|l}
\hline DOI: $10.1159 / 000350064$ & C 2013 S. Karger AG, Basel
\end{tabular}

Published onlıne: AprII 09, 2013

www.karger.com/kbr

Tadzic/Mihalj/Vcev/Ennen/Tadzic/Drenjancevic: Endocan, Endothelial Cell Adhesion Molecules and their Ligands Expression in Hypertensive Patients

ICAM-1. Conclusions: The increased expression of ICAM/VACM ligands, together with decrease of SCAMs and endocan suggests the de-activation of endothelium with reduction in $\mathrm{BP}$, decreasing the adherence of circulatory leukocytes to endothelium; subsequently decreasing the risk for development of atherosclerosis.

Copyright $@ 2013$ S. Karger AG, Basel

\section{Introduction}

Hypertension, diabetes mellitus, obesity, lipid metabolism dysregulation, smoking and manifested cardiovascular diseases (heart failure, myocardial ischemia) are condition with underlying endothelial dysfunction, increased oxidative stress and development of vascular complications [1-5]. Perturbation of endothelial function underlies the atherosclerotic process $[6,7]$. Endothelial adherence and migration of leukocytes into tissue is mediated by different sets of adhesion molecules expressed on activated endothelial cells and their complementary ligands on leukocytes, such as ICAM-1:LFA1 pair, or VCAM-1:VLA4 pair [8]. The expression of these sets might not only preselect the types of leukocytes that enter the inflammatory sites, but also activate these leukocytes, induce adherence to epithelial cells, and cause the release of cytokines, which lead to inflammatory response and endothelial damage, such as in atherosclerosis [7], lung diseases [9] and diabetes mellitus [10]. In atherosclerosis, ICAM-1 is up-regulated in sites prone to atherosclerosis development [11, 12], basically due to endothelium activation and inflammatory response. This is necessary step for recruitment of inflammatory cells, release of cytokines and adsorbance of the lipids into the atherosclerotic plaque [8]. When over-expressed on the activated endothelial cells, ICAM- 1 and VCAM-1, among others, undergo shedding and their soluble forms, sICAM- 1 and sVCAM-1 respectively, are detectable in serum and considered to be markers of endothelial cell activity or injury [13]. Leukocyte ligands for ICAM-1 and VCAM-1 are Lymphocyte Function-associated Antigen-1 (CD11a/CD18, alphaL/beta2 integrin; LFA-1) and Very Late Antigen-4 (CD49d/CD29, alpha4/beta1 integrin; VLA-4) respectively and they participate in cell recruitment to sites of inflammation, as well as in multiple immune cell interactions. Integrin-dependent cell adhesion can be rapidly and reversibly modulated in response to cell signaling [14]. Newly emerging data demonstrated that in responses to turbulent flow, dyslpidemia or some other stimuli (such as increase in intraluminal pressure $[15,16]$, endothelial cells over-express adhesion molecules. In addition, in subjects newly diagnosed with hypertension with no secondary cause, serum concentration of sE-selectin, sP-selectin and sICAM- 1 and some other markers of inflammation are significantly elevated compared to normotensive subjects $[17,18]$.

Although extensively investigated in inflammation and tumorigenesis [12, 14, 19], as well as in atherosclerosis $[6-8,11]$, almost none is known about the circulatory leukocytes interaction with endothelial cells in essential hypertension. Rare studies such as study by Ianone et al [13], in patients with autoimmune disease systemic sclerosis (SSc) having pulmonary hypertension (PAH) have demonstrated that peripheral blood T-cells expressing LFA-1 were significantly higher in patients with pulmonary hypertension (SSc-PAH) compared to patients with SSc without PAH. The proportion of T cells bearing VLA-4 antigen was significantly reduced in the SSc-PAH group compared to healthy controls and compared to patients with SSc without pulmonary hypertension. Expression of L-selectin on T cells was significantly lower in patients with SSc-PAH than in the healthy control group or in patients with SSc without PAH [13]. In addition, in patients with SSc-PAH, serum soluble ICAM-1, VCAM-1, P-selectin and PECAM-1 levels were higher than in healthy controls. All together, this study suggests that changes in the T cell/endothelium interplay take place in pulmonary hypertension in patients with systemic sclerosis, at least.

Endocan (ESM-1, the proteoglycan), is secreted by endothelial cells [20]. It has been shown to compete with ICAM-1 for LFA-1 [21]. Endocan circulates in plasma of healthy subjects and is increased in acute and severe inflammation [20], while endocan concentration is decreased in obese persons [6]. Thus, endocan can be considered as a marker of endothelial 


\section{Kidney \\ Blood Pressure Research}

Kidney Blood Press Res 2013;37:103-115

\begin{tabular}{l|l}
\hline DOI: $10.1159 / 000350064$ & C 2013 S. Karger AG, Basel
\end{tabular}

Published onlıne: April 09, 2013

www.karger.com/kbr

Tadzic/Mihalj/Vcev/Ennen/Tadzic/Drenjancevic: Endocan, Endothelial Cell Adhesion Molecules and their Ligands Expression in Hypertensive Patients

activation and its interaction with LFA-1 could be important in leukocyte adhesion and interaction with activated endothelium. Whether endocan is enhancing or preventing that interaction remains to be clarified.

The hypothesis of this study was that increased blood pressure is associated with higher serum sCAMs concentration and fewer free accessible circulatory leukocyte ligands for CAMs, since the adhesion of leukocytes and initiation of inflammatory process involves interaction of CAMs with their complementary ligands at leukocytes. Additional hypothesis is that this molecular interaction might involve endocan - mediated pathway, which is also a marker of endothelial activation. Therefore, normalization of blood pressure de-activates the endothelium, alleviates the inflammation process and changes the CAMs-ligands interaction. Subsequently, this could decrease the risk for development of atherosclerosis.

The aim of this study was to determine the effect of arterial blood pressure (BP) reduction on soluble cell adhesion molecules' (sICAM-1, VCAM-1 and E-selectin) and endocan plasma concentration; and the expression of their respective ligands (CD11a/LFA1, CD15/VLA-4 and CD49d) on circulatory leukocyte subpopulations in newly discovered hypertensive patients.

\section{Materials and Methods}

\section{Subjects}

Twenty four newly discovered hypertensive subjects of both sexes (age: $53 \pm 8$ yrs) were recruited from people who underwent health-screening examinations at Gesundheitszentrum Lange Reihe Dr. Tadzic und Kollegen, Hamburg, Germany. The inclusion criteria were patients with essential hypertension (systolic $\mathrm{BP} \geq 140 \mathrm{mmHg}$, and diastolic $\mathrm{BP} \geq 90 \mathrm{mmHg}$ ). The exclusion criteria were: secondary forms of hypertension, autoimmune disease, angina pectoris, coronary heart disease, myocardial infarction, cerebrovascular disease, haemoragic stroke, ischaemic stroke including transient ischemic attack and renal and liver disease.

After initial clinical assessment (bellow), blood pressure measurement and blood sampling, patients were administered Ca-channel blocker, amlodipin (5-10 mg/day for 8 weeks; dose to reach BP $\leq 139 / 89$ $\mathrm{mmHg}$. Peripheral blood samples were taken prior of administration of the antihypertensive drug and again after 8 weeks of continuous therapy. Age, sex, body weight, body mass index (BMI), blood pressure measurement, waist and hip circumference (to calculate waist. to hip ratio), and laboratory parameters (fasting glycaemia, high-density lipoprotein (HDL) and low-density lipoprotein (LDL) cholesterol, triglycerides, plasma renin activity (PRA), aldosterone, and urinary sodium, potassium, creatinine and urea concentration, were recorded for each patient at the first visit before of starting the therapy and 8 weeks after the therapy. The results are presented in Table 1. The study was approved by the Ethical Committees of the University Josip Juraj Strossmayer Osijek, Faculty of Medicine and by the Ethical Committee of the Gesundheitszentrum Lange Reihe Dr. Tadzic und Kollegen. All patients voluntary participated in the study and provided written informed consent.

The serum soluble cell adhesion molecules (sCAMs) ICAM-1, VCAM-1, E-selectin and endocan concentrations measurement

As endothelial activation markers, serum concentration of soluble vascular cell adhesion molecule (sVCAM)-1, soluble intercellular adhesion molecule (sICAM)-1 and sE-selectin were assessed by commercially available ELISA kit (eBioscience, Platinum ELISA, Austria), while endocan (ESM1) plasma concentration was assessed by ESM1 ELISA kit (USCN, Life Science Inc., China). Normal serum values from healthy controls determined by the manufacturers are as follows: s(VCAM)-1 772.4 2207.2 (range 400.6 $1340.8 \mathrm{ng} / \mathrm{ml}$ ), s(ICAM-1) 504 $\pm 171 \mathrm{ng} / \mathrm{ml}$ (range 302 - $1115 \mathrm{ng} / \mathrm{ml}$ ), sE-selectin 66.5 \pm 34.8 (range 21.0 - $186.0 \mathrm{ng} / \mathrm{ml}$ ). Data for endocan were no available. Leukocyte activation markers and soluble endothelial markers were assessed at baseline and 8 weeks after the antihypertensive therapy with Ca-channel blocker amlodipin (5-10 mg/day for 8 weeks; dose to reach BP $\leq 139 / 89 \mathrm{mmHg}$ ). 


\section{Kidney Blood Pressure Research}

Kidney Blood Press Res 2013;37:103-115

DOI: 10.1159/000350064

Published onIIne: Aprit 09, 2013

(C) 2013 S. Karger AG, Basel

www.karger.com/kbr

idzic/Mihalj/Vcev/Ennen/Tadzic/Drenjancevic: Endocan, Endothelial Cell Adhesion Molecules and their Ligands Expression in Hypertensive Patients
Table 1. The anthropometric and laboratory data the patients that participated in the study $(\mathrm{N}=24)$

\begin{tabular}{|c|c|c|c|}
\hline Parameters & Normal value & Baseline & $\begin{array}{l}\text { After 8-Weeks Th } \\
\text { (amlodipine) }\end{array}$ \\
\hline Age (years) & $\mathrm{N} / \mathrm{A}$ & $53.4 \pm 7,9$ & $53.6 \pm 8,1$ \\
\hline Body weight $(\mathrm{kg})$ & - & $90.3 \pm 14.6$ & $91.2 \pm 14.5$ \\
\hline BMI $\left(\mathrm{kg} / \mathrm{m}^{2}\right)$ & - & $31.4 \pm 4.9$ & $32.091 \pm 4.7$ \\
\hline waist to hip ratio (WHR) & - & $0.93 \pm 0.07$ & $0.95 \pm 0.07$ \\
\hline Systolic BP (mmHg) & $<139$ & $155 \pm 7$ & $134 \pm 13^{*}$ \\
\hline Diastolic BP( $\mathrm{mmHg})$ & $<89$ & $98 \pm 3$ & $84 \pm 12 *$ \\
\hline Total cholesterol (mg/dl) & $<220$ & $212 \pm 26$ & $213 \pm 33$ \\
\hline HDL cholesterol $(\mathrm{mg} / \mathrm{dl})$ & $>60.00$ & $52 \pm 12$ & $46 \pm 12$ \\
\hline LDL cholesterol $(\mathrm{mg} / \mathrm{dl})$ & $<100$ & $134 \pm 27$ & $139 \pm 32$ \\
\hline Triglycerides (mg/dl) & $<150$ & $162,19 \pm 54,53$ & $186 \pm 90$ \\
\hline Glucose $(\mathrm{mmol} / \mathrm{l})$ & $3.9-5.5$ & $6.1 \pm 0.5$ & $6.3 \pm 1.2$ \\
\hline PRA (nmol/L/h) & $1.68-23$ & $16.21 \pm 32.60$ & $39.65 \pm 48.38 *$ \\
\hline Aldosteron (mg/l) & $10-160$ & $83.10 \pm 29.77$ & $159.91 \pm 97.52 *$ \\
\hline Urea (mmol/L) & $2.5-7.5$ & $5,33 \pm 1.47$ & $6.25 \pm 1.87$ \\
\hline Creatinine (mmol/L) & $0.55-1.1$ & $0.78 \pm 0.16$ & $0.79 \pm 0.16$ \\
\hline $\mathrm{Na}^{+}$serum $(\mathrm{mmol} / \mathrm{L})$ & $137-145$ & $138.83 \pm 1.72$ & $140.15 \pm 2.13^{*}$ \\
\hline $\mathrm{K}^{+} \operatorname{serum}(\mathrm{mmol} / \mathrm{L})$ & $3.6-5.0$ & $4.13 \pm 0.44$ & $3.93 \pm 0.31 *$ \\
\hline
\end{tabular}

Basic blood analysis

Patients` blood was sent to the routine diagnostic laboratory at Dres, Ennen und Gebauer, Hamburg (Germany) immediately after sampling. Serum levels of HDL, LDL, total cholesterol, triglycerides, glucose, creatinine, sodium and potassium were determined by Abbott architect c16000 blood analysis instrument. Levels of aldosterone and renin were determined by RIA (Siemens, Los Angeles, USA).

Assessment of CD11a/LFA-1, CD15 and CD49d expression on blood circulatory leukocytes

Antibodies and reagents. The human sE-selectin Platinum Elisa (BMS 205 / BMS205TEN, the human sVCAM-1 BMS232/BMS232TEN, the human sCD105 BMS2105INST and the human sICAM-1 BMS201/ BMS201 TEN) were obtained from eBioscience (Vienna, Austria). Endocan (endothelial-cell specific molecule - 1 (ESM1-1) antibodies were obtained from USCNK Life Science Inc. The antibodies used for flow cytometry were phycoerythrin conjugates purchased from eBioscience, as follows: anti-CD11a (clone HI111); anti-CD15 (clone HI98) and antiCD49d (clone 9F10).

Blood collection and processing for flow cytometry. Patients' blood was collected into tubes containing EDTA (Becton Dickinson, San Diego, CA). $100 \mu \mathrm{l}$ of whole blood was added to FACS tube and stained according to the manufacturers' protocol (eBioscience, Protocol B: Human Lysed Whole Blood). In short, the cells were incubated with phycoerythrin (PE) - conjugated primary antibodies (anti-CD11a, anti-CD15 or antiCD49d) or their appropriate isotype controls at 1:100 dilution for 20 minutes on RT in the dark. The erythrocytes were lysed by using 1xRBC lysing buffer (eBioscience).

Flow cytometry data analysis. Dead cells were excluded based on their light scattering properties. At least 20,000 live cells were collected by a BD FACSCalibur cytometer and analyzed using the WinMDI software. Monocytes, granulocytes and lymphocyte were identified and gated on the sideward versus forward scatter plot and further analyzed for CD11a, CD15 and CD49d expression on a histogram. Expression data are presented as the geometric mean of fluorescence intensity determined by subtracting geometric mean fluorescence intensity of the isotype control from geometric mean fluorescence intensity of the specific mAb.

\section{Statistical analysis}

The sample size required to show a significant effect on sCAMs in hypertensive patients, was calculated based on preliminary data in 10 patients with alpha $=0.05$ and a statistical power of $80 \%$ for t-test and ANOVA showing a needed sample size of 17 patients for sCAMs and other biochemical parameters, while for leukocyte ligands, the sample size was determined as 10 patients. Shapiro Wilk test was used to test the data distribution. For normally distributed data, dependant variables were compared by paired t-test, independent variables by Student's t-test and the difference between leukocytes ligands expression among the cell subpopulations by One-way ANOVA followed by Tukey post-hoc test. In the case of abnormal data distribution Mann-Whitney U-test, Wilcoxon`s test, and Kruskal-Wallis analysis of variance test followed by 


\section{Kidney Blood Pressure Research}

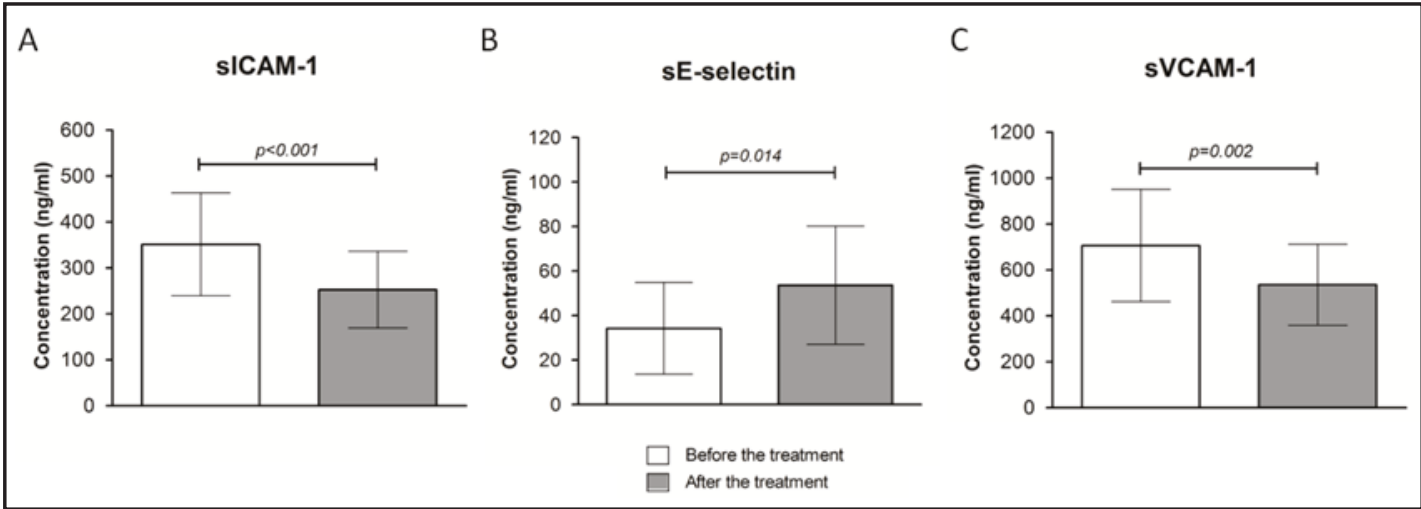

Fig. 1. The plasma soluble cell adhesion molecules (sCAMs): ICAM-1 (panel A), E-selectin (panel B) and VCAM-1 (panel C) concentrations before (white bars) and after (gray bars) the normalization of blood pressure with amlodipine therapy. Data are expressed as mean \pm SD for $\mathrm{N}=24$ patients; p- value of each relation is expressed at the panels.

Dunn`s post-hoc test were employed. In addition, Pearson's and Sperman`s correlation were calculated in the case of normally and abnormally distributed data, respectively. All calculations were performed with software SigmaPlot v.11. All data are presented as mean \pm SD. $p \leq 0.05$ was considered significant.

\section{Results}

\section{Subjects}

The anthropometric and laboratory data of patients ( $\mathrm{N}=24 ; 13$ men and 11 women) that conformed to inclusion and exclusion criteria and sucessfuly participated in the study are presented in the Table 1 . The average age of patients was $53 \pm 8$ yrs. All patients were obese (BMI $\left.>30 \mathrm{~kg} / \mathrm{m}^{2}\right)$ with hyperlipidemia, hyperglycaemia and hypertension at the time of enrollement. After 8 weeks of amlodipin therapy (dose: 5-10 mg/day for 8 weeks; to reach $\mathrm{BP} \leq 139 / 89 \mathrm{mmHg}$ ), there was significant decrease in systolic and diastolic blood pressure, with values bellow 139/89 mmHg. PRA and aldosterone levels significanlty increased, while urine sodium concentration significanlty increased and urine potassium concentration significanlty decreased with the treatment. There were no significant changes in plasma urea and creatinine values (Table 1).

The serum soluble cell adhesion molecules (sCAMs) ICAM-1, VCAM-1, E-selectin and endocan concentrations

Serum levels of sICAM-1 (351.4 \pm 111.9 vs. $252.9 \pm 83.6 ; \mathrm{p} \leq 0.001)$ and sVCAM-1 (706.6 \pm 244.5 vs. $535.5 \pm 176.8 ; \mathrm{p}=0.002)$ were significantly decreased after the treatment, compared to control conditions, while E-selectin levels were significantly increased after the amlodipin treatment $(34.3 \pm 20.56$ vs $53.6 \pm 26.6, \mathrm{P}=0.014)$ (Figure 1$)$. Endocan levels tended to decrease with BP reduction $(\mathrm{p}=0.063)$ (Figure $2 \mathrm{~A})$.

CD11a/LFA-1, CD15 and CD49d expression on blood circulatory leukocytes

Expression of CD11a/LFA-1 (ICAM-1 and endocan ligand) was significantly increased in all three cell types with BP decrease (Ly $p=0.009$, Mo $\mathrm{p} \leq 0.001$, Gr p=0.005). CD15 (E-selectin ligand) showed weak reactivity on lymphocytes and monocytes, but it was abundant on granulocytes. CD15 and CD49d/VLA-4 (VCAM-1 ligand) did not change significantly after the treatment. All three ligands showed significantly different pattern of expression on various cell types $(\mathrm{p} \leq 0.001)$ (Figures 3C). Gating strategy and representative histograms are shown in Figure $3 \mathrm{~A}$ and $3 \mathrm{~B}$, respectively. There was no significant correlation between any of the CAMs-ligand pair. 


\section{Kidney Blood Pressure Research}

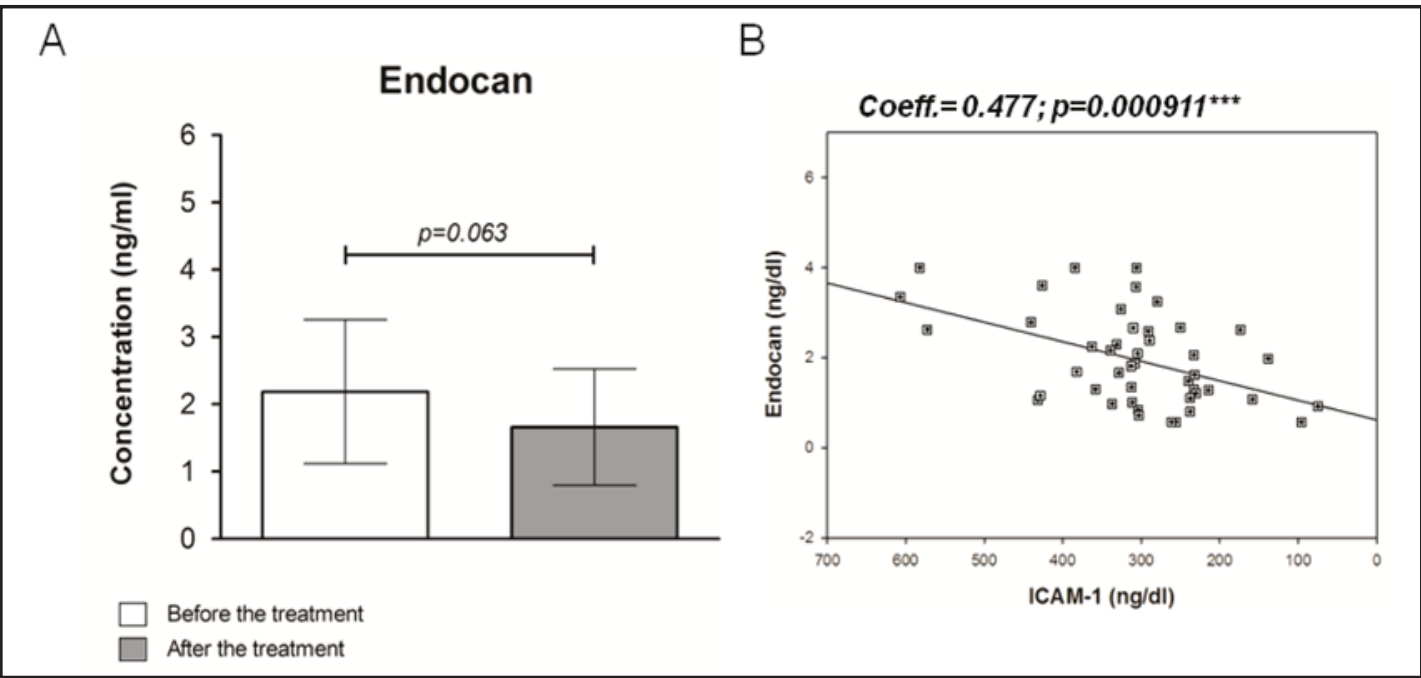

Fig. 2. The serum endocan concentration before (white bar) and after (gray bar) the amlodipine therapy is shown at Panel A. Panel B shows significant positive correlation of plasma sICAM-1 and endocan concentration. Data are expressed as mean \pm SD for $\mathrm{N}=24$ patients; 45 results are plotted on the graph, $\mathrm{p}$ value of each relation is expressed at the panels.

However, there was significant positive correlation of systolic and diastolic BP with ICAM-1 and VCAM-1 (Figure 4A and 4B, respectively), and negative correlation of systolic BP with their respective leukocyte ligands, reaching significance for granulocyte and monocyte CD11a/LFA-1 (Figure 5). Endocan significantly positively correlated with ICAM-1 (Figure 2B). In addition, there was no significant correlation between the degree of blood pressure reduction $(\triangle \mathrm{BP})$ and the degree of adhesion molecules changes ( $\triangle \mathrm{CAMs}$ ) for any of the adhesion molecules, except in the case of $\Delta$ sVCAM-1 and $\Delta$ diastolic BP, where a significant negative correlation was found $(\mathrm{p}=0.0339, \mathrm{r}=-0.545$; Figure $4 \mathrm{C})$.

\section{Discussion}

The cell adhesion molecules, such as ICAM-1, VCAM-1 and E-selectin just recently emerged as an important marker of endothelial activation preceding the adhesion of the activated leukocytes and initiating the atherosclerotic lesions. Endothelial dysfunction is in core of hypertension [7, 22, 23], effects of high dietary salt intake [22, 23] and diabetes mellitus [7]. However the understanding of the role of inflammation and endothelialleukocyte interaction in subsequent vascular damage in these conditions is at its beginnings and mainly investigated in the field of autoimmune diseases [13].

The novelty of this study is the attempt to inter-relate the markers of endothelial cell activation, their corresponding ligands on circulatory leukocytes and blood pressure, and to elucidate the possible contribution of the endocan pathway in development of initial conditions for adherence of activated leukocytes to endothelium in hypertensive patients prior to and after the blood pressure reduction.

The major findings in this study are the following: a) significantly decreased serum levels of sICAM-1 and sVCAM-1 with arterial blood pressure reduction after amlodipin treatment (Figure 1); b) with decrease in blood pressure, the expression of CD11a/LFA-1 (ICAM-1 and endocan ligand) was significantly increased in all three leukocyte cell types (lymphocytes, monocytes and granulocytes) while CD15 and CD49d/VLA-4 (VCAM-1 ligand) did not change significantly (Figure 3A-C, respectively); c) There was significant positive correlation of systolic and diastolic BP with ICAM-1 and VCAM-1, and negative correlation of systolic BP of 


\section{Kidney Blood Pressure Research}

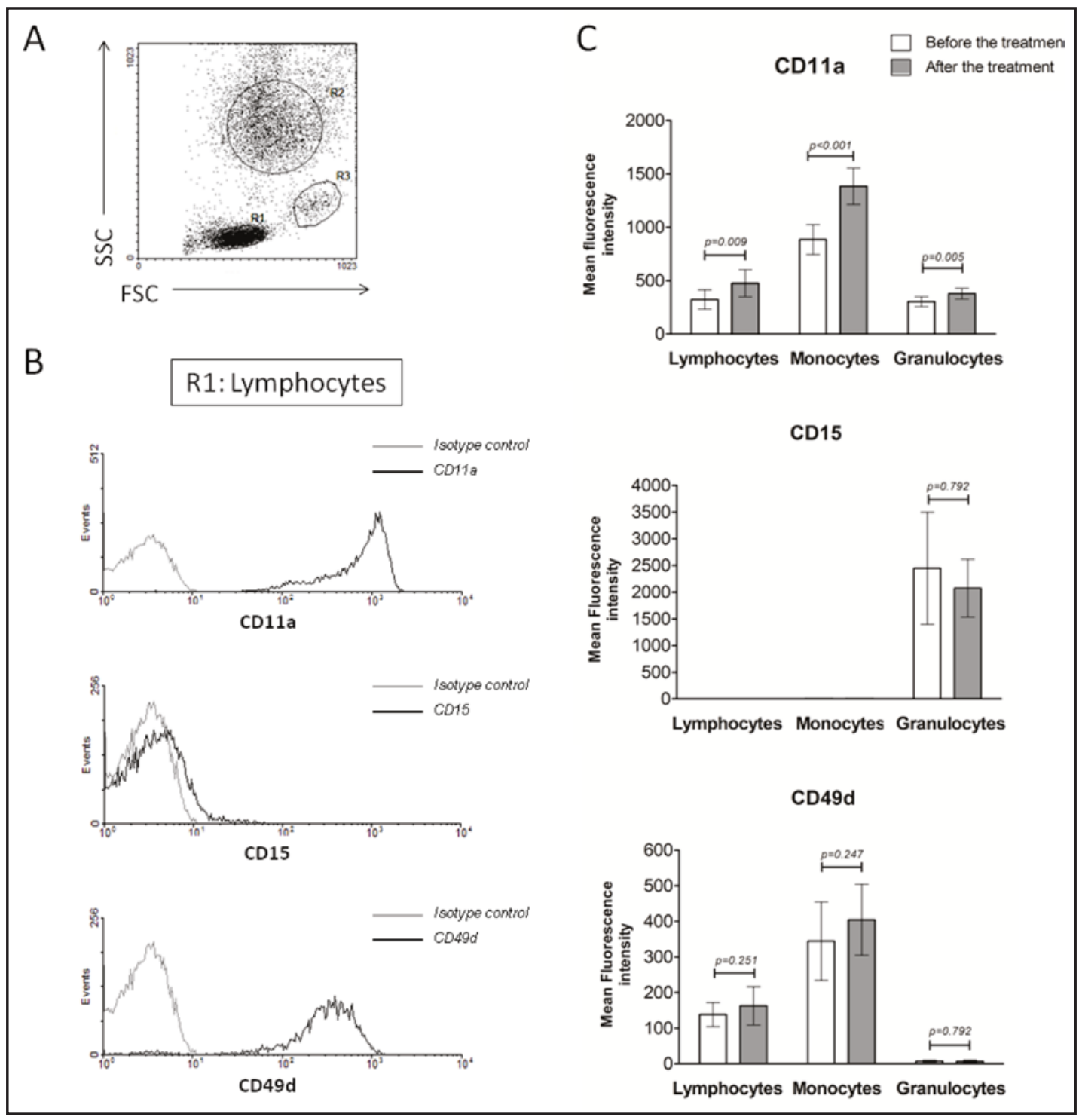

Fig. 3. Level of ICAM-1, VCAM-1 and E selectin ligands was assessed by flow cytometry, and their expression presented as the geometric mean of fluorescence intensity determined by subtracting geometric mean fluorescence intensity of the isotype control from geometric mean fluorescence intensity of the specific $\mathrm{mAb}$. The representative side scatter versus forward scatter plot defining leukocytes subpopulations (R1 - lymphocytes, R2 - granulocytes, R3 - monocytes) is shown on Panel A. Panel B shows representative histograms for determination of CD11a/LFA-1 (ICAM-1 and endocan ligand), CD15 (E-selectin ligand) and CD49d/VLA-4 (VCAM-1 ligand) expression on lymphocytes (R1 gate on panel A). Panel C shows different pattern of expression of CD11a, Cd15 and CD49d on the lymphocytes, monocytes and granulocytes before (white bar) and after (gray bar) the amplodipin treatment. All three ligands showed different pattern of expression on various cell types (Panel $\mathrm{C} ; \mathrm{p} \leq 0.001) .(\mathrm{N}=10$ patients, before and after the therapy).

ICAM-1 leukocyte ligands, LFA-1 for granulocytes and monocytes (Figure 4A, 4B and Figure 5 respectively); d) endocan positively correlated with ICAM-1 and tended to decrease with $\mathrm{BP}$ reduction (Figure $2 \mathrm{~B}$ and $2 \mathrm{~A}$, respectively).

The adhesion of leukocytes via CAMs and their complementary ligands at leukocytes involves the inflammation mediated by TNF- $\alpha$ signaling pathway, which has been shown to instigate the VCAM-1 and ICAM-1 expression in the endothelium [24]. In atherosclerosis, 


\section{Kidney Blood Pressure Research}

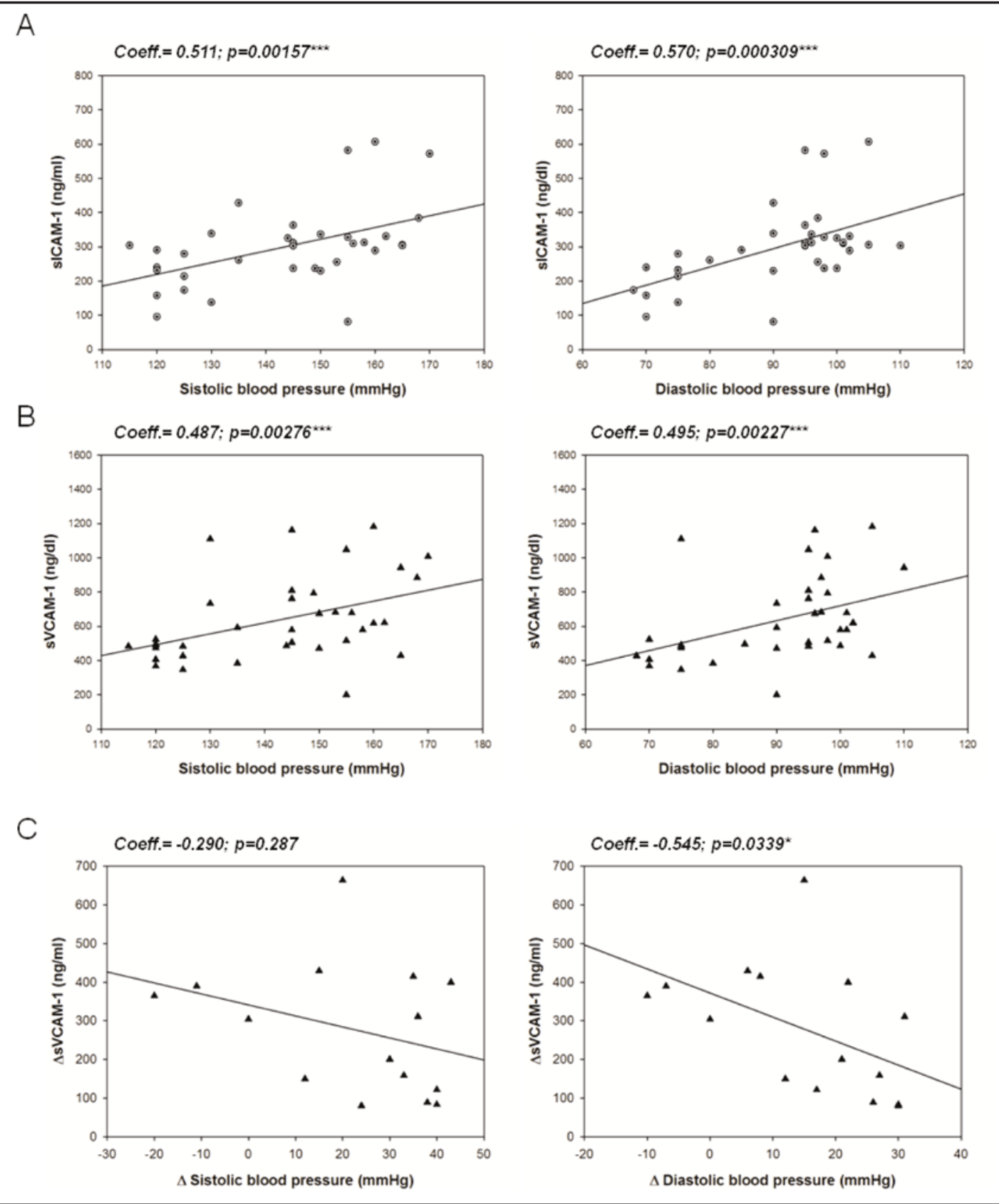

Fig. 4. Correlation between soluble cell adhesion molecules concentration with systolic and diastolic blood pressures - sICAM-1 and systolic (A, left) and diastolic (A, right) blood pressure; and VCAM-1 and systolic (B, left) and diastolic (B, right) blood pressure. In addition, correlation between the degree of blood pressure reduction $(\triangle \mathrm{BP})$ and the degree of sVCAM-1 change $(\triangle \mathrm{sVCAM}-1)$ is shown on panel $\mathrm{C}$. There is a significant positive correlation of both sCAMs with BP, and a significant negative correlation between $\Delta$ sVCAM-1 and $\Delta$ diastolic BP. p value of each relation is expressed at the panels. 36 resuts are plotted at the pannels $\mathrm{A}$ and $\mathrm{B}$, and 17 at the panel $\mathrm{C}$.

ICAM-1 is up-regulated in sites prone to atherosclerosis development [11, 12]. Our finding that sICAM-1 and SVCAM-1 are significantly decreasing with decrease in blood pressure (endothelial deactivation) is in agreement of previous findings that VCAM-1 is not expressed in intact endothelium; however, its expression is significantly increased in atherosclerosis 


\section{Kidney Blood Pressure Research}

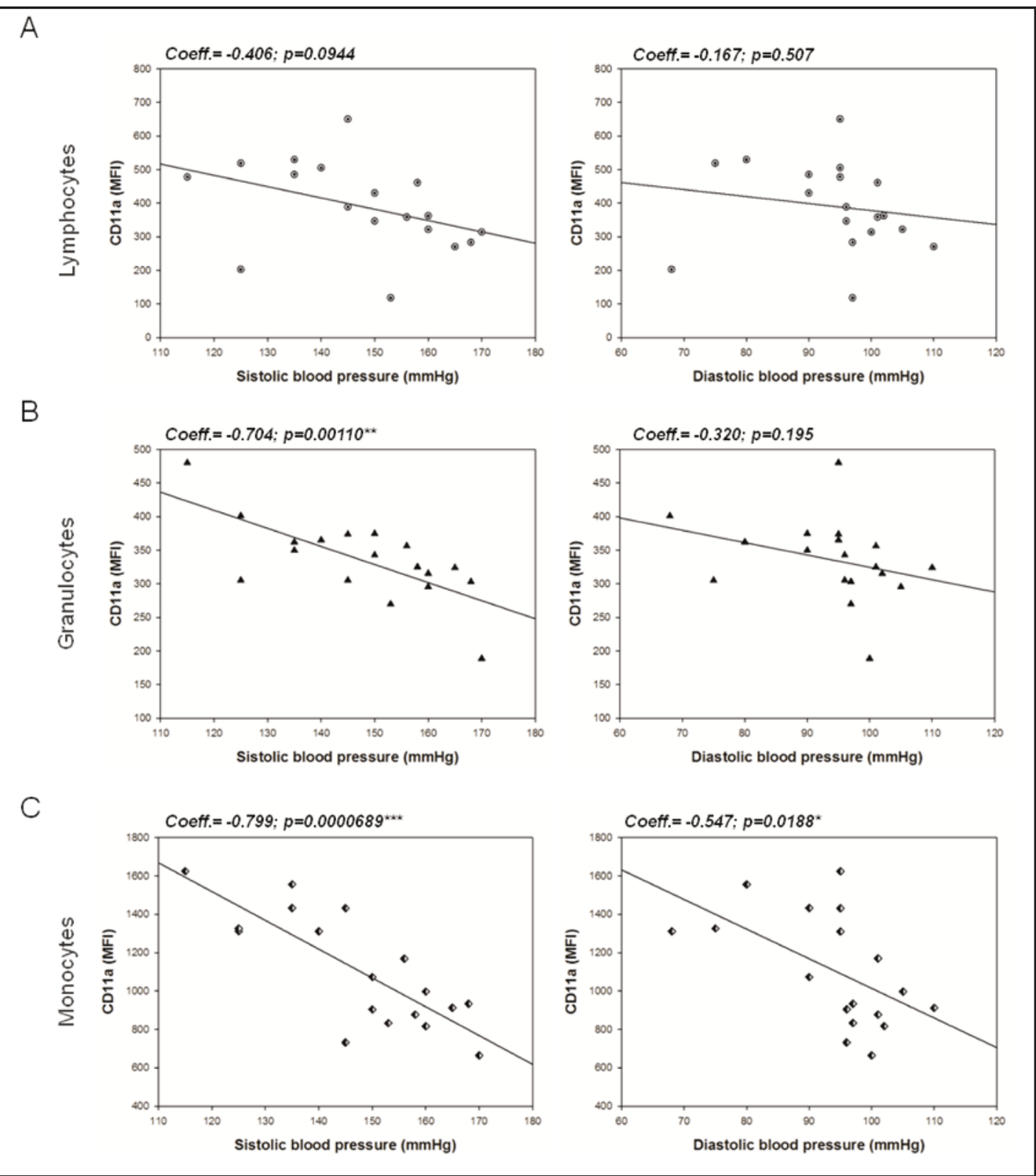

Fig. 5. Correlation between CD11a/LFA-1 expression on peripheral blood lymphocytes (A), granulocytes (B) and monocytes (C) with systolic (left panels) and diastolic blood pressures (right panels). There is a significant positive correlation of CD11a with BP; $p$ value of each relation is expressed at the panels; 18 resuts are plotted at each graph.

[11]. ICAM-1 expression is stimulated with proinflammatory cytokines TNF- $\alpha, I L-1 \beta, I L-4$ or INF $\gamma[25,26]$. It mediates monocyte infiltration and could be important in proliferation of vascular smooth muscle cells [25-27]. Or vice versa, in hypertension, in SHR rats, there is a significant increase in sICAM-1, sVCAM-1 $[18,27]$. Interestingly, sE-selectin showed different pattern of expression in response to changes in blood pressure - it increases with blood pressure reduction. E-selectin is expresssed only on cytokines-activated endothelium and facilitates neutrophils' recruitment . Neutrophils' tethering and rolling on E-selectin, upon exiting the microvasculature, down-regulate their surface L-selectin through ectodomain shedding by a disintegrin and metalloprotease 17 (ADAM17) [28], E-selectin is associated with the development of acute coronary syndrome [29]. As a marker of endothelial 


\section{Kidney Blood Pressure Research}

Kidney Blood Press Res 2013;37:103-115

\begin{tabular}{l|l}
\hline DOI: 10.1159/000350064 & (C) 2013 S. Karger AG, Basel
\end{tabular}

Published onlıne: Aprit 09, 2013

www.karger.com/kbr

Tadzic/Mihalj/Vcev/Ennen/Tadzic/Drenjancevic: Endocan, Endothelial Cell Adhesion Molecules and their Ligands Expression in Hypertensive Patients

activation, E-selectin has been shown to be elevated in hypertension [18]. Rubio-Guerra et al evaluated the relationship between the levels of circulating soluble CAMsand the degree of atherosclerosis in hypertensive type- 2 diabetic patients and found significant correlations between ICAM-1 levels and maximal carotid artery intimal-medial thickness in these patients. No correlation was observed with E-selectin and VCAM-1. These results suggest that ICAM1 , but not E-selectin is associated and correlated with the degree of atherosclerosis in type-2 diabetic hypertensive patients [7]. Interestingly, increased levels of sE-selectin have been found in hypotensive patients with sepsis while other markers of endothelial activation (ICAM-1, VCAM-1) remained unchanged [30]. This is in agreement with E.selectin increase with blood pressure reduction as demonstrated in present study (Figure 1). In addition, CD15 (Syalyl Lewis ${ }^{\mathrm{x}}$, E-selectin ligand) expression did not vary significantly after the treatment. Sialyl Lewis ${ }^{\mathrm{x}}$ is constitutively expressed on granulocytes and monocytesand on activated $\mathrm{T}$ and $\mathrm{B}$ lymphocytes [31]. Taken together, our findings suggest that with decrease in blood pressure, there is a de-activation of the endothelial cells, followed by changes in the adhesion of the circulatory leukocytes. ICAM-1 and its ligand LFA-1 is the most prominent pair to mediate the interaction of endothelium with leukocytes while blood pressure is high.

Endocan (ESM-1, the proteoglycan), is secreted by endothelial cells. It has been shown to compete with ICAM-1 for LFA-1 [21] thus possibly inhibiting leukocytes adhesion and transmigration [21]. The results of present study of tendency of endocan concentration to decrease and significant positive correlation of endocan with ICAM-1 (Figure 2B) would be in agreement with study by Bechard et al [21]. Considering the crucial role of the ICAM-1/LFA1 interactions during firm adhesion of human lymphocytes and monocytes to endothelium [21] endocan may be considered as a protective and limiting in the regulation of monocyte/ granulocytes extravasation at the inflammatory sites or at the activated endothelium in preventing exhaustive adhesion of the leukocytes and possible subsequent damage of the endothelium. This potential mechanism is schematically represented and explained in Figure 6.

VLA-4 and LFA-1 integrins belong to a large family of adhesion receptors widely expressed on immune cells $[32,33]$. They participate in cell recruitment to sites of inflammation, as well as multiple immune cell interactions [14]. New emerging studies demonstrated that increase in endothelial NO/cGMP changes, i.g. decreases the affinity of VCAM-1 to VLA-4, having de-adhesive effects [34].

One intriguing finding of this study is the increased expression of leukocyte adhesion ligands with the decrease in blood pressure (Figure 3). The expression of CD11a/LFA-1 (ICAM-1 and endocan ligand) was significantly increased in all three leukocyte cell types (lymphocytes, monocytes and granulocytes) after amlodipin treatment. One possible explanation for that would be that with the blood pressure normalization, endothelial cells deactivate and release previously attached leukocytes. This is in agreement with study by Mills et al [34] that in response to exercise, hypertensive subjects showed significantly greater mean density of CD11a on lymphocytes and on monocytes, two times greater increased in IL-2 and increased adhesion of peripheral blood monocytes to endothelial cell layer compared to normotensive subjects. Furthermore, in normotensive subjects, the adhesion of leukocytes decreased following exercise. Taken together, in hypertension, there is endothelial activation and increased mononuclear cell adhesion to endothelial cells, possibly through cytokineinduced activation of mononuclear cell CD11a. This may be relevant to the increased risk of atherosclerosis in human hypertension [35]. Additional evidence for our hypothesis of deactivation of the endothelium and de-activation and/or de-adhesion of leukocytes is finding that infusion of isoprotenerol, non-specific beta-adrenergic agonist increases the number of circulating CD62Llow/CD11ahigh leukocytes more in hypertensive than in normotensive subjects. In addition, in hypertensive subjects the sICAM-1 concentration was elevated compared to normotensive subjects [36].

These findings are further supported by the population study on association of hypertension with inflammation, endothelial dysfunction and abnormal metabolism among Mongolian people in China [18]. Rates of abnormal metabolism, elevated CRP, elevated sICAM-1, elevated sE-selectin and elevated angiotensin II as well as coexistence of 


\section{Kidney \\ Blood Pressure Research}

Fig. 6. Shematic presentation of the potential mechanism of blood pressure reduction on cell adhesion molecules' interaction with their ligands on circulatory leukocyte subpopulation, and the potential counter-balancing role of endocan. In hypertension, there is high concentration of ICAM-1 and VCAM-1, with low E-selectin on endothelium, as markers of endothelial cell activation. This is accompanied with high adherence of leukocytes via LFA-1 and VLA-4 interaction with endothelium. Endocan, released by activated endothelium competes with ICAM-1 for LFA-1 thus disturbing the leukocyte adherence (hypothetical protective effect of endocan; left
Kidney Blood Press Res 2013;37:103-115

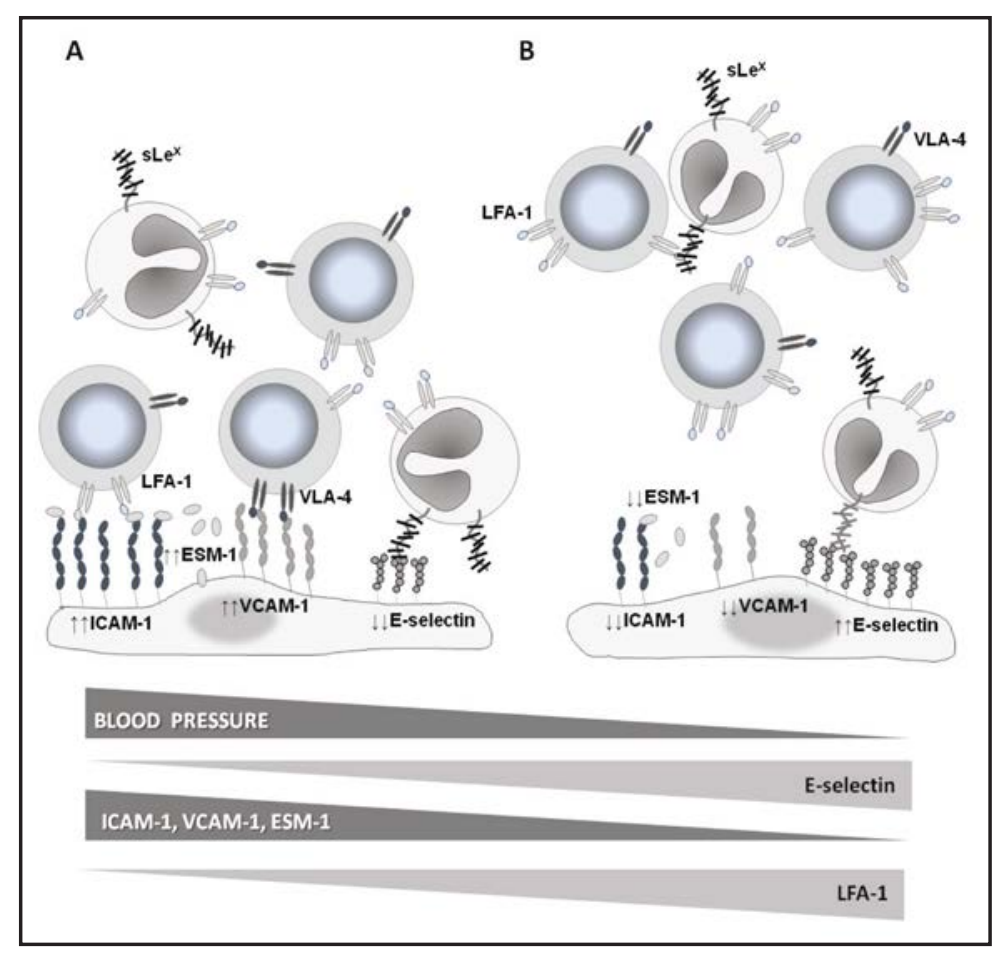

side of the Figure 6). With blood pressure reduction, there is decrease in ICAM-1, VCAM-1 expression and concomitant increase in the expression of their ligands LFA-1 and VLA-4 on possibly detached leukocytes, accompanied by the decrease in endocan secretion (right side of the Figure 6). Endocan, released by activated endothelium competes with ICAM-1 for LFA-1 thus disturbing the leukocyte adherence (hypothetical protective effect of endocan; left side of the Figure 6). With blood pressure reduction, there is decrease in ICAM-1, VCAM-1 expression and concomitant increase in the expression of their ligands LFA-1 and VLA-4 on possibly detached leukocytes, accompanied by the decrease in endocan secretion (right side of the Figure 6).

abnormal metabolism with the elevated biomarkers were all higher in hypertensive than in normotensive subjects indicating that individuals with co-existence of abnormal metabolism with inflammation and endothelial dysfunction had higher risk of prevalent hypertension [18].The limitation of the present study is our inability to directly measure the adherent leukocytes to endothelium in our patients. For example, intra-vital microscopy is an excellent method to study in vivo leukocyte recruitment and adhesion and has been used in various models of experimental animals and in cell cultures [37, 38]. However, it is not suitable for in vivo studies in humans at all.

Based on the results of the present study, it is possible to propose the potential mechanism of blood pressure reduction on cell adhesion molecules interaction with their ligands on circulatory leukocyte subpopulation and the potential counter-balancing role of endocan, as shown in Figure 6. In hypertension, there is high concentration of sICAM-1 and s VCAM-1, with low sE-selectin levels, as markers of endothelial cell activation. This is accompanied with high adherence of leukocytes via LFA-1 and VLA-4 interaction with endothelium. Endocan, released by activated endothelium could compete with ICAM-1 for LFA-1 thus disturbing the leukocyte adherence (hypothetical protective effect of endocan; left side of the Figure 6). With blood pressure reduction, there is decrease in ICAM-1, VCAM1 expression and concomitant increase in the expression of their ligands LFA-1 and VLA-4 on possibly detached leukocytes, accompanied by the slight decrease in endocan secretion (right side of the Figure 6). In addition, Ca-channels antagonists per se have been shown to reduce the intima-media thickness progression in hypertension and slowed the progression of early coronary atherosclerosis [39]. 


\section{Kidney \\ Blood Pressure Research}

Kidney Blood Press Res 2013;37:103-115

\begin{tabular}{l|l}
\hline DOI: $10.1159 / 000350064$ & C 2013 S. Karger AG, Basel
\end{tabular}

Published onIIne: Aprit 09, 2013

www.karger.com/kbr

\section{Conclusion}

Tadzic/Mihalj/Vcev/Ennen/Tadzic/Drenjancevic: Endocan, Endothelial Cell Adhesion Molecules and their Ligands Expression in Hypertensive Patients

In hypertension, endocan could be secreted by activate endothelium and might have protective role by competing with ICAM-1 for their ligand LFA-1 on to-be-adherent leukocytes. The increased expression of sCAMs' ligands on circulatory leukocytes, together with significant decrease of endothelial CAMs after amlodipin treatment suggests the de-activation of the endothelium with successful reduction in blood pressure. This could subsequently decrease the risk for development of atherosclerotic lesions.

\section{Conflict of Interests}

None of the authors has any conflicts in relation to the work presented in this paper.

\section{References}

-1 Panza J, Quyumi A, Brush J, Epstein S: Abnormal endothelium-dependent vascular relaxation in patients with essential hypertension. N Engl J Med 1990;323:22-27.

2 Vogel R, Coretti M, Plotnick G: Changes in flow-mediated brachial artery vasoactivity with lowering of desirable cholesterol levels in healthy middle-aged man. Am J Cardiol 1996;77:37-40.

- 3 Celermajer DS, Adams MR, Clarkson P: Passive smoking and impaired endothelium-dependent arterial dilatation in healthy young adults. N Engl J Med 1996;334:150-154.

4 Goodfellow J, Ramsey MW, Luddington LA: Endothelium and inelastic arteries: an early marker of vascular dysfunction in non-insulin dependent diabetes. Br Med J 1996;312:744-745.

-5 Tawakol A, Omland T, Gerhard M, Wu JT, Creager MA: Hyperhomocyst(e)inemia is associated with impaired endothelium-dependent vasodilatation in humans. Circulation 1997;95:1119-1121.

6 Janke J, Engeli S, Gorzelniak K, Feldpausch M, Heintze U, Böhnke J, Wellner M, Herse F, Lassalle P, Luft FC, Sharma AM: Adipose tissue and circulating endothelial cell specific molecule-1 in human obesity. Horm Metab Res 2006;38:28-33.

-7 Rubio-Guerra AF, Vargas-Robles H, Serrano AM, Vargas-Ayala G, Rodriguez-Lopez L, Escalante-Acosta BA: Correlation between the levels of circulating adhesion molecules and atherosclerosis in hypertensive type2 diabetic patients. Clin Exp Hypertens 2010;32:308-310.

8 Crowther MA: Pathogenesis of atherosclerosis. Hematol Am Soc Hematol Educ Program 2005:436-441.

-9 Popper HH, Pailer S, Wurzinger G, Feldner H, Hesse C, Eber E: Expression of adhesion molecules in allergic lung diseases. Virchows Arch 2002;440:172-180.

10 Bacun T, Glavas-Obrovac L, Belovari T, Mihaljević I, Hanich T, Belaj VF, Vcev A: Insulin administration in the mild hyperglycaemia changes expression of proinflammatory adhesion molecules on human aortic endothelial cells. Coll Antropol 2010;34:911-915.

11 Nakashima Y, Raines EW, Plump AS, Breslow JL, Ross R: Upregulation of VCAM-1 and ICAM-1 at atherosclerosis-prone sites on the endothelium in the ApoE-deficient mouse. Arterioscler Thromb Vasc Biol 1998;18:842-851.

12 Frank PG, Lisanti MP: ICAM-1: role in inflammation and in the regulation of vascular permeability. Am J Physiol 2008;295:H926-H927.

13 Iannone F, Riccardi MT, Guiducci S, Bizzoca R, Cinelli M, Matucci-Cerinic M, Lapadula G: Bosentan regulates the expression of adhesion molecules on circulating T cells and serum soluble adhesion molecules in systemic sclerosis-associated pulmonary arterial hypertension. Ann Rheum Dis 2008;67:1121-1126.

14 Chigaev A, Sklar LA: Aspects of VLA-4 and LFA-1 regulation that may contribute to rolling and firm adhesion. Front Immunol DOI: 10.3389/fimmu.2012.00242.

15 Riou S, Mees B, Esposito B, Merval R, Vilar J, Stengel D, Ninio E, van Haperen R, de Crom R, Tedgui A, Lehoux S: High pressure promotes monocyte adhesion to the vascular wall. Circ Res 2007;100:1226-1233.

16 Michell DL, Andrews KL, Woollard KJ, Chin-Dusting JP: Imaging Leukocyte Adhesion to the Vascular Endothelium at High Intraluminal Pressure. J Vis Exp 2011;54:pii3221.

17 Kavita K. Shalia, Manoj R. Mashru, Jagdish B. Vasvani, Rajashree A. Mokal, Shilpa M. Mithbawkar, Priti K: Thakur Circulating levels of cell adhesion molecules in hypertension. Indian J Clin Biochem 2009;24:388397. 


\section{Kidney \\ Blood Pressure Research}

Tadzic/Mihalj/Vcev/Ennen/Tadzic/Drenjancevic: Endocan, Endothelial Cell Adhesion Molecules and their Ligands Expression in Hypertensive Patients

18 Zhang M, Wang G, Wang A, Tong W, Zhang Y: Association of hypertension with coexistence of abnormal metabolism and inflammation and endothelial dysfunction. Blood Press DOI: 10.3109/08037051.2012.745226.

19 Perut F, Cenni E, Unger RE, Kirkpatrick CJ, Giunti A, Baldini N: Immunogenic properties of renal cell carcinoma and the pathogenesis of osteolytic bone metastases. Int J Oncol 2009;34:1387-1393.

20 Bechard, D, Meignin V, Scherpereel A, Oudin S, Kervoaze G, Bertheau P, Janin A, Tonnel AB, Lassalle P: Characterization of the secreted form of endothelial-cell-specific molecule 1 by specific monoclonal antibodies. J Vasc Res 2000;37:417-425.

21 Béchard D, Scherpereel A, Hammad H, Gentina T, Tsicopoulos A, Aumercier M, Pestel J, Dessaint JP, Tonnel AB, Lassalle P: Human endothelial-cell specific molecule-1 binds directly to the integrin CD11a/CD18 (LFA-1) and blocks binding to intercellular adhesion molecule-1. J Immunol 2001;167:3099-3106.

-22 Drenjančević-Perić I, Jelaković B, Lombard JH, Kunert MP, Kibel A, Gros M: High-salt diet and hypertension: focus on the Renin-Angiotensin system. Kidney Blood Press Res 2011;34:1-11.

-23 Drenjancevic-Peric I, Frisbee JC, Lombard JH: Skeletal muscle arteriolar reactivity in SS BN13 consomic rats and Dahl salt-sensitive rats. Hypertension 2003;41:1012-1015.

24 Sethi G, Sung B, Aggarwal BB: TNF: a master switch for inflammation to cancer. Front Biosci 2008;13:50945107.

25 Acton ST, Wethmar K, Ley K: Automatic tracking of rolling leukocytes in vivo. Microvasc Res 2002;63:139148.

26 Hartung A: Lipid-basierte Transfektionssysteme für die posttranskriptionelle Expressionshemmung von endothelialem VCAM-1 als potentiell antiinflammatorischer Therapieansatz. MathematischNaturwissenschaftlichen Fakultät der Rheinischen Friedrich-Wilhelms-Universität Dissertation, 2011 URN:urn:nbn:de:hbz:5N-26113. Bonn, April 2011.

27 McCarron RM, Wang L, Sirén AL, Spatz M, Hallenbeck JM: Monocyte adhesion to cerebromicrovascular endothelial cells derived from hypertensive and normotensive rats. Am J Physiol 1994;267:H2491-H2497.

28 Fang F, Zhang W, Yang L, Wang Z, Liu DG: PECAM-1 and E-selectin expression in vulnerable plague and their relationships to myocardial Leu125Val polymorphism of PECAM-1 and Ser128Arg polymorphism of E-selectin in patients with acute coronary syndrome. Zhonghua Xin Xue Guan Bing Za Zhi 2011;39:11101116.

29 Schaff U, Mattila PE, Simon SI, Walcheck B: Neutrophil adhesion to E-selectin under shear promotes the redistribution and co-clustering of ADAM17 and its proteolytic substrate L-selectin. J Leukoc Biol 2008;83:99-105.

-30 Schuetz P, Jones AE, Aird WC, Shapiro NI: Endothelial cell activation in emergency department patients with sepsis-related and non-sepsis-related hypotension. Shock 2011;36:104-108.

31 Liu FR, Jiang CG, Li YS, Li JB, Li F: Cimetidine inhibits the adhesion of gastric cancer cells expressing high levels of sialyl Lewis $\mathrm{x}$ in human vascular endothelial cells by blocking E-selectin expression. Int J Mol Med 2011;27:537-544.

-32 Dixit N, Yamayoshi I, Nazarian A, Simon SI: Migrational Guidance of Neutrophils Is Mechanotransduced via High-Affinity LFA-1 and Calcium Flux. J Immunol 2011;187:472-481.

-33 Sarantos MR, Zhang H, Schaff UY, Dixit N, Hayenga HN, Lowell CA, Simon SI: Transmigration of neutrophils across inflamed endothelium is signaled through LFA-1 and Src family kinase. J Immunol 2008;181:86608669.

-34 Chigaev A, Smagley Y, Sklar LA: Nitric oxide/cGMP pathway signaling actively down-regulates alpha4beta1integrin affinity: an unexpected mechanism for inducing cell de-adhesion. BMC Immunol 2011;12:28.

35 Mills PJ, Farag NH, Perez C, Dimsdale JE: Peripheral blood mononuclear cell CD62L and CD11a expression and soluble interstitial cell adhesion molecule-1 levels following infused isoproterenol in hypertension. J Hypertens 2002;20:311-316.

-36 Mills PJ, Maisel AS, Ziegler MG, Dimsdale JE, Carter S, Kennedy B, Woods VL Jr: Peripheral blood mononuclear cell-endothelial adhesion in human hypertension following exercise. J Hypertens 2000;18:1801-1806.

37 Granger DN, Kubes P: The microcirculation and inflammation: modulation of leukocyte-endothelial cell adhesion. J Leukoc Biol 1994;55:662-675.

38 Spicer A, Calfee CS: Fixing the leak: targeting the vascular endothelium in sepsis. Crit Care 2012;16:177.

-39 Hernández RH, Armas-Hernández MJ, Velasco M, Israili ZH, Armas-Padilla MC: Calcium antagonists and atherosclerosis protection in hypertension. Am J Ther 2003;10:409-414. 